\title{
Reports of Meetings
}

Neuroepidemiology 1991;10:223

Notices of Meetings

Activity International Tropical Neurology Congress

Place Limoges, France

Date $\quad$ September 26-28, 1991

Topics Neuroepidemiology, Retroviruses, Malaria, Neuromuscular diseases

Language $\quad$ English,ffirench (simultaneous translation)

Contact Prof. Michel Dumas

AddressM Institut de Neurologie Tropicale, Faculty de M6decine, rue du Dr. Marcland, 87025 Limoges, France

Travel agency Bernis Tourisme, 24 Place de la R6publique, 8JZ0D0 Limoges, France

Activity

Place

Date

Topics

Language

Contact

Address

International Course of Neuroepidemiology

Buenos Aires, Argentina

October 2-3, 1991

Field studies, Clinical trials, Stroke, Epilepsy, Parkinson, Dementia, Infections

English, Spanish (simultaneous translation)

Dr. Manuel J. Somoza

Av. del Libertador $22546^{\circ} \mathrm{D}, 142^{\wedge}$ Buenos Aires, Argentina

Activity

Place

Date

Topics

Langu $^{\wedge} \mathrm{S}$

Contact

Address

5th Panamerican Symposium of Neuroepidemiology

Montevideo, Uruguay

October 5, 1991

Field studies, Stroke, Epilepsy, Infections, Tropical neurology

English, Spanishj^nultaneous translation)

Dr. Gustavo Roman 
Federal Building 714, NIH, Bethesda, MD 20892, USA

Activity

Place

8th Panamerican Congress of NeufMogy

Date Montevideo, Uruguay

Topics

October 6-12, 1991

lobe, DementiaSymposia Neuroimaging, Parkinson and movement disorders, Epilepsy, Cognitive

evoked

potentials, Sleep disorders, Education and research in neurologyLanguageEnglish, Spanish (simultaneous

translation)

Contact

Dr. Carlos Chouza

Address

Congrexpo, 18 de Julio 1791, Of. 101, Montevideo, Uruguay

Activity

Place

19th Intern^Kmal Epilepsy Congress

Date

Rio de Janeiro, Brazil

Topics

October 14-19, 1991

Rehabilitation, Quality of life, Tropical diseases, NeurocysticercosisSymposia $\quad$ Neurobiology of epilepsy,

Epilepsy surgery, Clinical trials of new antiepileptic drugs,

Clinical relevance of the epidemiology of epilepsyLanguage English, Portuguese, Spanish (simultaneous translation)

Contact Dr. P. Rog6rio M. de Bittencourt, ggcretary General

A. Address Congrex do Brasil

Rua do Ouvidor 60 - Grupo 414

20040 Rio de Janeiro

RJ

Brazil

Travel agency Host Turismo de Eventos

Rua Sao Clemente 407

22260 Rio de Janeiro

RJ

Brazil 